\title{
Pharmacological treatment of fibromyalgia
}

\begin{abstract}
Worldwide, fibromyalgia (FM) has increased its prevalence in recent years, becoming a public health problem. Despite the advances in research, this pathology remains uncertain, although several theories are known that try to explain its etiology and pathophysiology, the most accepted are focused on a multifactorial alteration, which causes a variation in different stages of the pain path both in the central and peripheral area. It lacks an objective method for its diagnosis and a therapeutic target, the result is an ineffective and insecure treatment, causing a negative impact on the quality of life of the sufferer and its environment, producing high costs in the health system. The main objective of this study is to describe the pharmacological management of FM, for which a descriptive, observational, retrospective study was carried out, based on the review of 75 medical records, in the integrated subnet of southern health services in Bogotá, finding prescriptions not in accordance with the European League against Rheumatism (EULAR) 2016 guide, given by the formulation of opioids, gabapentin, in monotherapy or dual therapy and triple with risky interactions, which are not indicated in this pathology due to their high risk, with Low profit and high costs. It is recommended to start with non-pharmacological management; When this is required, it should be performed individually based on the associated symptoms, not on pain, and also refrain from the formulation of combined treatments, since its long-term efficacy and safety are unknown.
\end{abstract}

Keywords: fibromyalgia, pathophysiology, drug treatment, safety, costs
Volume 8 Issue I - 2020

\author{
Diana Yisenia Pinzón Fajardo,' Jose Julián \\ Lopez Gutiérrez,' Jorge Mario Camacho \\ Carvajal $^{2}$ \\ 'Faculty of Science, Pharmacy Department, National University \\ of Colombia, Colombia \\ ${ }^{2}$ Epidemiologist doctor and master in bioethics
}

\author{
Correspondence: Diana Yisenia Pinzón Fajardo, Faculty \\ of Science, Pharmacy Department, National University of \\ Colombia, cross $85 \mathrm{~N}^{\circ} 54-19$ flat 509 building 5 conjunto 8 , \\ Bogotá, Colombia, Tel 3003830984, \\ Email dypinzonfa@unal.edu.co
}

Received: December 19,2019 | Published: January 07, 2020

\section{Introduction}

Fibromyalgia (FM) is a complex and little known disease, health professionals face a pathology with a difficult diagnosis, without a therapeutic target, which causes a high burden of morbidity and costs. ${ }^{1-4}$ There is no single treatment that has proven effective. Several attempts have been made to elucidate the best ways to diagnose and treat FM, but so far no objective evidence has been found to allow the diagnosis to be made or ruled out. ${ }^{1,5-7}$ Functional magnetic resonance imaging and biochemical marker tests are not useful because they indicate the existence of pain, but not its cause. ${ }^{8,9}$ This leads to the diagnosis being controversial because it is based only on subjective criteria, which can cause a misdiagnosis of the disease, since each doctor can give a different interpretation has shown that not all doctors know the criteria from the American College of Rheumatology (ACR) to establish a diagnosis. ${ }^{10-12}$ This is sometimes because many doctors do not accept FM as a pathology, and consider it as a poor adaptive response to stress or depression, increasing patient frustration and generating poor adherence to medical management, which leads to rupture of the doctor-patient relationship. One of the contradictors of the FM is Dr. Frederick Wolfe, director of the National Data Bank for Rheumatic Diseases and lead author of the 1990 article that first defined the diagnostic guidelines for FM, who considers that it is not a disease if not a physical response to stress, depression and economic and social anxiety. Wolfe said that "some of us in those days thought that we had actually identified a disease, which clearly is not," "Making people sick, making them sick, was something wrong." Therefore, FM is considered not It requires no pharmacological treatment and that this has only been driven by the profits produced by the sale and commercialization of some medications as without being clear about how it reduces pain. ${ }^{5,11}$

Several studies (clinical trials, meta-analyzes and systematic reviews) have failed to demonstrate which is the best treatment, since they report lack of efficacy in the control of symptoms, therefore, the different management guidelines differ in their recommendations and are based on expert opinion. The latest guidelines change vision by recommending non-pharmacological management as the first line of treatment and recommend pharmacological treatment only to control the associated symptoms, which may have an impact on the severity and prognosis of FM. ${ }^{1,6,13-15}$

It is necessary to generate pharmacoepidemiological evidence on the management of FM, since it is not only a complex pathology that is characterized by pain, which indicates suffering, but also generates an important economic burden for the health system and for the patient. A study that characterizes our population and evaluated the appropriate prescription of medications allows us to show a real picture of the prescription used in FM, the mistakes made in the formulation and the safety of the medications used in this population.

This research is the first step to generate different types of studies, which allows evaluating the safety and effectiveness of different pharmacological and non-pharmacological treatments, such as clinical studies or cost-effectiveness and / or cost-benefit studies. These future investigations can help to better understand this disease, and sensitize health professionals, that although it is not a pathology that has high mortality, if it is a generator of high morbidity which causes a significant deterioration of the patient, limiting his working life, social and family, not forgetting the economic impact, the direct, indirect and intangible costs that this pathology has for the health system and who suffers from it.

\section{Material and methods}

Design is supported by a study of the use of prescriptiontype medicines, which uses a descriptive observational design of longitudinal cut with retrospective collection. This study presents three analytical processes. The first is to show the evolution of fibromyalgia with pharmacological treatment, describing the changes in the associated symptoms, in the numerical pain scale, and in the formulation. Following up from January 2016 to December 2017, in patients who attend an outpatient clinic of the southern health network. 
The second is to carry out a sociodemographic characterization and a description of the pathological history of people diagnosed with fibromyalgia who come to medical care in the southern subnet in Bogotá. A total of 1,745 clinical records were received, of which 75 met the inclusion criteria and did not present exclusion criteria. Which were people over 18 years of age, with a diagnosis of fibromyalgia within their clinical history, pain scale, associated symptoms and drug treatment. Two time cuts were taken, the first one was called pharmacological treatment $\mathrm{N}^{\circ}$, the variables to be evaluated were taken and the second time cut was pharmacological treatment $\mathrm{N}^{\circ} 2$ where changes in these variables were evaluated compared to the first treatment.

And finally, the cost of the pharmacological treatment formulated to the 75 patients was made for the time that the study was carried out, for this the direct costs were calculated from the perspective of the health system, and each drug was dug in the database of the Sismed filtering by national, laboratory and wholesaler, the value per minimum, average and maximum milligram was calculated in the $\mathrm{R}$ program of each medication. These values were entered in the Excel database and calculated by the number of patients formulated for each medication, the dose formulated, the treatment time and with these data the average follow-up was obtained in months, average consumption of each medication in a month and the cost of a patient in a month and the total cost of all patients which was divided into those formulated with monotherapy, dual therapy and triple therapy. The values were calculated in Colombian pesos.

\section{Results}

Of the total population, $93 \%$ belong to the female gender, with a woman-to-man ratio of $14 / 1$, predominantly young and old adults. The majority of the population are married followed by singles. The specialties that $70 \%$ fibromyalgia patients are managing are pain clinic, followed by $20 \%$ orthopedics.

\section{Medicines formulated in treatment No. I}

Acetaminophen 54.6\% (41/75) predominated, followed by pregabalin 42.7\% (32/75), tramadol 24\% (18/75), Amitriptyline $22.6 \%(17 / 75)$, acetaminophen codeine or hydrocodone $22.6 \%$ (17/75), gabapentin $1.3 \%(1 / 75)$, naproxen $2.7 \%(2 / 75)$ and fluoxetine $1.3 \%(1 / 75)$. This formulation was given on average for 9.8 months.

\section{Changes regarding treatment No. 2}

Duloxetine formulation increased by $18.6 \%(14 / 75)$, acetaminophen plus codeine increased 13\% (10/75), pregabalin increased $13 \%$ (10.75), gabapentin increased 2.6\% (2/75), acetaminophen increased $1.3 \%(1 / 75)$, hydrocodone increased $1.3 \%(1 / 75)$, amitriptyline decreased $28 \%$ (21/75), tramadol decreased 14\% (11/75), Fluoxetine and naproxen were suspended and paroxetine and tapentadol were first formulated in treatment No. 2 .

\section{Adverse reactions}

Adverse reactions reported in $20 \%(\mathrm{n}=15 / 75)$. The predominant ones were at the level of the CNS (drowsiness, dizziness, headache), gastrointestinal (nausea, vomiting, diarrhea, epigastralgia) and at the cardiovascular level (tachycardia). The medications associated with these symptoms are acetaminophen, amitriptyline, duloxetine, pregabalin and tramadol.

\section{Psychiatric diseases}

They were not reported in 40\% (30/75), anxiety disorder and depression 34\% (26/75), depressive disorder 28\% (21/75), Adjustment disorder $8 \%(6 / 75)$, Traits badly adaptive personality $6 \%(5 / 75)$, posttraumatic stress $4 \%(3 / 75)$. An antecedent is not exclusive of others, since a patient can present several symptoms.

\section{Numerical pain scale}

$55 \%$ of the patients presented an increase in pain, $24 \%$ did not report any change and $21 \%$ presented a decrease in pain in 1 to 2 units. Therapeutic failure of $79 \%$ of patients.

\section{Associated symptoms}

Insomnia 66\% (50/75), sadness $42 \%(30 / 75)$, anxiety $29 \%(22 / 75)$, diarrhea or constipation $26 \%$ (20775), memory loss $16 \% / 12 / 75)$ and fatigue $14 \%(11 / 75)$. One symptom is not exclusive to others, since a patient may have several symptoms.

\section{Costs of treatment as fibromyalgia monotherapy}

Table 1. Costs per patient of the medications used in fibromyalgia, are the costs of one patient for each medication in a month. Source: own calculations from the database of the medical records of the southern sub network of Bogotá.

Table I Costs of treatment as fibromyalgia monotherapy

\begin{tabular}{lllllll}
\hline $\begin{array}{l}\text { No. } \\
\text { Patients }\end{array}$ & Monotherapy & Average tracking & $\begin{array}{l}\text { Mg consumption } \\
\text { per patient }\end{array}$ & $\begin{array}{l}\text { Cost Month per } \\
\text { patient }\end{array}$ & $\begin{array}{l}\text { Cost Month per } \\
\text { patient }\end{array}$ & $\begin{array}{l}\text { Cost Month per } \\
\text { patient }\end{array}$ \\
\hline 3 & Medicine & Months & Month & Average & Minimum & Maximum \\
\hline 15 & Hydrocodone & 6 & 450 & $\$ 54,138$ & $\$ 113,751$ & $\$ 192,685$ \\
2 & Codeine & 4 & 563 & $\$ 6,750$ & $\$ 15,694$ & $\$ 28,913$ \\
10 & Naproxen & 7 & 38077 & $\$ 8,964$ & $\$ 11,052$ & $\$ 14,579$ \\
1 & Duloxetine & 6 & 96 & $\$ 4,593$ & $\$ 9,186$ & $\$ 10,651$ \\
18 & Duloxetine & 9 & 9000 & $\$ 4,606$ & $\$ 5,746$ & $\$ 7,808$ \\
42 & Tramadol & 5 & 2700 & $\$ 2,450$ & $\$ 2,980$ & $\$ 3,575$ \\
1 & Acetaminophen & 4 & 44758 & $\$ 537$ & $\$ 1,835$ & $\$ 4,118$ \\
32 & Fluoxetine & 3 & 600 & $\$ 1,317$ & $\$ 1,590$ & $\$ 1,788$ \\
17 & Pregabalin & 4 & 209 & $\$ 874$ & $\$ 1,275$ & $\$ 1,687$
\end{tabular}




\section{Costs of dual fibromyalgia therapy}

Table 2. Cost of dual therapy of fibromyalgia patients costs were calculated for one patient and for the total number of patients who were formulated as dual therapy, as shown in the table and the total is given for the management of 22 patients. Source: own calculations from the database of the medical records of the southern sub network of Bogotá.

\section{Costs of triple fibromyalgia therapy}

Table 3. Cost of triple therapy for fibromyalgia patients costs were calculated for one patient and for the total number of patients who were formulated as triple therapy, as shown in the table and the total is given for the management of 22 patients. Source: own calculations from the database of the medical records of the southern sub network of Bogotá

Table 2 Costs of dual fibromyalgia therapy

\begin{tabular}{|c|c|c|c|c|c|c|}
\hline \multirow{2}{*}{ Dual } & \multirow[b]{3}{*}{ Medicine } & \multicolumn{5}{|l|}{ Month cost } \\
\hline & & \multicolumn{3}{|l|}{ Patient } & \multicolumn{2}{|l|}{ Total Patients } \\
\hline No. Patients & & minimum & medium & Minimum & Minimum Total & Maximum Total \\
\hline I & Duloxetine + Tramadol & $\$ 7,044$ & $\$ 12,166$ & $\$ 14,226$ & $\$ 7,044$ & $\$ 14,226$ \\
\hline I & Acetaminophen + Duloxetine & $\$ 5,130$ & $\$ 11,021$ & $\$ 14,769$ & $\$ 5,130$ & $\$ 14,769$ \\
\hline 1 & Amitriptyline + Gabapentin & $\$ 4,687$ & $\$ 5,839$ & $\$ 7,921$ & $\$ 4,687$ & $\$ 7,921$ \\
\hline 7 & Acetaminophen + Tramadol & $\$ 2,988$ & $\$ 4,815$ & $\$ 7,693$ & $\$ 20,913$ & $\$ 53,850$ \\
\hline 2 & Pregabalin + Tramadol & $\$ 3,325$ & $\$ 4,254$ & $\$ 5,262$ & $\$ 6,650$ & $\$ 10,524$ \\
\hline 6 & Acetaminophen + Pregabalin & $\$ 1,4 \mid I$ & $\$ 3,110$ & $\$ 5,805$ & $\$ 8,468$ & $\$ 34,828$ \\
\hline I & Amitriptyline + Tramadol & $\$ 2,531$ & $\$ 3,073$ & $\$ 3,688$ & $\$ 2,531$ & $\$ 3,688$ \\
\hline I & Acetaminophen + Amitriptyline & $\$ 618$ & $\$ 1,928$ & $\$ 4,231$ & $\$ 618$ & $\$ 4,231$ \\
\hline 2 & Amitriptyline+ Pregabalin & $\$ 955$ & $\$ 1,368$ & $\$ 1,800$ & $\$ 1,910$ & $\$ 3,600$ \\
\hline (n 22) $29 \%$ & Total & & & & $\$ 57,95 \mathrm{I}$ & $\$ 147,636$ \\
\hline
\end{tabular}

Table 3 Costs of triple fibromyalgia therapy

\begin{tabular}{|c|c|c|c|c|c|c|}
\hline \multirow{2}{*}{ Triple } & & \multicolumn{5}{|c|}{ Month cost } \\
\hline & & \multicolumn{3}{|l|}{ Patient } & \multicolumn{2}{|l|}{ Total Patients } \\
\hline $\begin{array}{l}\text { No. } \\
\text { Patients }\end{array}$ & Medicine & Minimum & medium & Maximum & Minimum Total & Maximum Total \\
\hline I & Acetaminophen + Hydrocodone + Duloxetine & $\$ 59,268$ & $\$ 124,772$ & $\$ 207,453$ & $\$ 59,268$ & $\$ 207,453$ \\
\hline 3 & Acetaminophen + Hydrocodone + Pregabalin & $\$ 55,550$ & $\$ 116,861$ & $\$ 198,489$ & $\$ 166,649$ & $\$ 595,468$ \\
\hline I & Acetaminophen + Codeine + Duloxetine & $\$ 11,880$ & $\$ 26,715$ & $\$ 43,681$ & $\$ 11,880$ & $\$ 43,681$ \\
\hline 3 & Acetaminophen + Codeine + Tramadol & $\$ 9,738$ & $\$ 20,509$ & $\$ 36,605$ & $\$ 29,213$ & $\$ 109,816$ \\
\hline I & Acetaminophen + Codeine + Fluoxetine & $\$ 8,604$ & $\$ 19,119$ & $\$ 34,818$ & $\$ 8,604$ & $\$ 34,818$ \\
\hline I & Amitriptyline + Acetaminophen + Codeine & $\$ 7,368$ & $\$ 17,622$ & $\$ 33,143$ & $\$ 7,368$ & $\$ 33,143$ \\
\hline 2 & Acetaminophen + Pregabalin + Tramadol & $\$ 3,862$ & $\$ 6,089$ & $\$ 9,380$ & $\$ 7,724$ & $\$ 18,759$ \\
\hline 2 & Amitriptyline + Pregabalin + Tramadol & $\$ 3,406$ & $\$ 4,347$ & $\$ 5,375$ & $\$ 6,811$ & $\$ 10,750$ \\
\hline I & Acetaminophen + Amitriptyline + Pregabalin & $\$ 1,492$ & $\$ 3,203$ & $\$ 5,918$ & $\$ 1,492$ & $\$ 5,918$ \\
\hline (n 22) $29 \%$ & Total & & & & $\$ 356,139$ & $\$ 1,302,827$ \\
\hline
\end{tabular}




\section{Total cost}

Table 4. Total cost of the fibromyalgia drug treatment for the total of patients. Source: own calculations from the database of the medical records of the southern sub network of Bogotá.

Table 4 Total cost

\begin{tabular}{lll}
\hline Therapy & Minimum Total & Maximum Total \\
\hline Monotherapy & $\$ 57,225$ & $\$ 133,855$ \\
Dual & $\$ 57,951$ & $\$ 147,636$ \\
Tripe & $\$ 356,139$ & $\$ 1,302,827$ \\
Total & $\$ 47,315$ & $\$ 1,584,318$
\end{tabular}

\section{Discussion of the results}

The results obtained in the characterization of the population, compared with other studies are consistent, since they showed similarities in the population adult diagnosed with FM, with a higher percentage in women, predominantly in young and old adults. ${ }^{16}$ The criteria used for the diagnosis of fibromyalgia are not known, since these data were not available in the medical history.

The most commonly used medication alone or in combination was acetaminophen, the actions of paracetamol (acetaminophen), in the modulation of the endogenous cannabinoid system and as a serotonin receptor agonist, it may be beneficial for the management of symptoms, although there is no direct evidence with regarding the use of paracetamol in FM. ${ }^{17}$ Its prescription was higher as an analgesic, the high prescription of this medicine, may be due to the fact that it is safe in the geriatric population, and is indicated for the management of chronic musculoskeletal pain. ${ }^{18}$

EULAR recommends it only in some cases, ${ }^{10,19}$ such as the association of fibromyalgia and rheumatologic diseases, a systematic review was performed with acetaminophen for the management of chronic pain due to osteoarthritis of the knee and hip, which showed that improvement that acetaminophen provides in pain and functionality is minimal and this effect does not differ from the dose, the adverse effects are not serious, but liver function should be monitored for the risks it presents compared to placebo "(reported liver abnormalities of $8 / 1000$ participants treated with placebo and 70/1000 participants treated with paracetamol (RR 3.79, 95\% CI 1.94 to 7.39)) " ${ }^{20}$ In this study its prescription was chronic and the dose was increased in revaluation with maximum doses of $3000 \mathrm{mg}$ per day and formulations for up to one year.

Regarding naproxen, its prescription was scarce and was not formulated in the final treatment, the NSAID formulation is contraindicated, for the management of FM, and is used in low doses and for short periods of time, due to its side effects. ${ }^{15}$ The opioids were formulated in both treatments of low potency (tramadol, codeine) and high potency (hydrocodone), they were always used accompanied by a co-analgesic. Tramadol was not used as monotherapy, it was associated with acetaminophen, pregabalin or amitriptyline, but the combination more used was with acetaminophen.

In dual therapy, the formulation was higher in the initial treatment with respect to the final treatment ( $14 \%$ vs. $2.6 \%$ respectfully), this phenomenon can occur due to the increase in pain, which caused the formulation to be changed for the second treatment, this association of tramadol with acetaminophen does not have studies that support its use in FM, the optimal dose and its long-term efficacy are unknown. ${ }^{10}$ The use of tramadol and acetaminophen as a co-analgesic can have a beneficial effect, since it not only exerts an effect on the mu $(\mu)$ receptor, because this pathway is altered, it also has a 5HT and noradrenaline reuptake inhibitory activity and the Acetaminophen acts on different targets of the pain pathway, this may cause synergism in its effect, some studies show effectiveness for the control of FM symptoms, EULAR recommends it in cases of non-response to other treatments and presence of severe pain (EULAR). ${ }^{21}$

Codeine and hydrocodone were always accompanied with acetaminophen; codeine was the most formulated, significantly increasing its prescription, which did not happen with tramadol and acetaminophen with hydrocodone. Opioids are not recommended for FM management due to several reasons: 1. They are not effective in FM, because the endogenous opioid activity is altered, due to the lack of binding of the $\mu$ receptor by the endogenous opioids in the pain path, ${ }^{7,22} 2$. The adverse effects of Opioids, such as mental confusion, sedation and cognitive deficit, these symptoms reinforce the psychological effects in patients with anxiety and depression 3. Its long-term use produces opioid-induced hyperalgesia and may develop greater pain sensitivity such as diffuse allodynia, ${ }^{21} 4$. Concerns about the tendency to abuse, ${ }^{10}$ the chronic use of opioids leads to tolerance, which is why higher doses are required to produce the same analgesic effect. ${ }^{21}$ The EULAR guide recommends tramadol level III evidence (at least one randomized controlled trial), although with a low consensus, its use may be justified in case of osteaoatrosis. ${ }^{14}$ For the other opioids EULAR makes a level D recommendation (recommendation against its use) ${ }^{7,8}$

Another opioid formulated in the final treatment was tapentadol although its formulation was scarce, this could be due to its double effect on the u receptor, and for inhibiting the reuptake of norepinephrine, in a double-blind study, it has an efficacy comparable to oxycodone, with better tolerance at the gastrointestinal level, but there are no studies of its effectiveness in FM, ${ }^{21}$ its use in the EULAR guide is not contemplated, due to its lack of safety and efficacy studies, therefore its use is not advised

FM antidepressants, such as tricyclic antidepressants and dual reuptake inhibitors, act in the descending pain pathway, the first management option is amitriptyline because it is the most studied for FM, a systematic review was performed in Cochrane to evaluate the use of neuropathic pain and FM finding that most studies are biased to support a beneficial effect, it is in FM only beneficial in a minority of patients reporting for FM "a necessary number to treat (NNT) from 4.6 (3.6 to 6.6).

The analysis showed that even when using these potentially biased data, only about $38 \%$ of the participants obtained benefits with amitriptyline and $16 \%$ with placebo; most of the participants did not achieve adequate pain relief. The potential benefits of amitriptyline were supported by a lower rate of absence of withdrawals due to lack of efficacy; $8 / 153(5 \%)$ withdrew due to lack of efficacy with amitriptyline and 14/119 (12\%) with placebo"in terms of safety refer the number needed to harm (NND) was 4.1 (CI of 95\%: 3.2 to 5.7) (58), the study does not report adverse events presented for the NNT. EULAR suggest that low doses are those that have the best benefits and obtained a high degree of consensus, ${ }^{23}$ in this study, a majority of $25 \mathrm{mg}$ per day was formulated, which conforms to the guide's recommendation in its dose, but its formulation was scarce and decreased further in the final treatment. Start with 17 patients 
and end with 7, the causes of these decisions are unknown, but it may be because the population mostly belong to the elderly and this medication is not recommended in the geriatric population for chronic use $\mathrm{e}^{24}$ or It could also be given for the contraindications of the medicine. ${ }^{14}$

Selective serotonin reuptake inhibitors such as fluoxetine and paroxetine use were scarce (prescribed to a patient), this may be because studies show that they are not superior to placebo versus the improvement of associated symptoms, but may Being beneficial when associated with depression or anxiety, EULAR does not recommend its use. ${ }^{10}$ IRSN serotonin and norepinephrine reuptake inhibitors, acts in the descending pathway of pain, also help modulate mood and insomnia, ${ }^{10}$ although this effect is marginal, ${ }^{25,26}$ a systematic review was conducted with the Duloxetine for the management of neuropathic pain, chronic pain and fibromyalgia, finding that with a dose of $60 \mathrm{mg}$ the pain is reduced in FM, with an NNT for an improvement of $50 \%$ the pain is 8

It is not possible to determine whether the dose of $20 \mathrm{mg}$. It is effective, but the $120 \mathrm{mg}$ dose is not more effective. Due to the association of pain with depression, studies are required to evaluate its effectiveness, NNT 6 reports mild adverse effects, the most reported are insomnia or drowsiness, constipation, headache, malaise, dry mouth or dizziness. ${ }^{27}$ In this study, duloxetine increased its formulation in the final treatment; it was found that patients who were initially treated with amitriptyline were changed to duloxetine or pregabalin, which would explain starting with a $13 \%$ predominance in monotherapy and ending with $32 \%$, with similar uses in the three prescription modalities. EULAR recommends its use when FM is associated with depression and anxiety, and the doses should be adjusted according to response and safety, the most used dose was $30 \mathrm{mg}$, which conforms to the EULAR guidelines, very little was formulated of $60 \mathrm{mg}$ Because this dose is not recommended by consensus experts, duloxetine is of choice in severe pain and sleep disorders. ${ }^{10}$ The use of anticonvulsants in FM, such as pregabalin and gabapentin act as membrane stabilizers, ${ }^{28,29}$ a systematic review of cochrane anticonvulsants in fibromyalgia was performed. The quantity and quality of the evidence were insufficient to establish definitive conclusions about the efficacy and safety of gabapentin, lacosamide, pregabalin and levetiracetam in FM.

Studies with pregabalin showed fewer biases, with greater reduction of NNT pain of 12 compared to placebo, did not significantly reduce fatigue, insomnia and the dropout rate was higher in pregabalin vs placebo due to adverse effects with a NND 13, dizziness it was the most frequent adverse event, ${ }^{30}$ the optimal dose is unknown, but it is postulated that it should be 300 to $450 \mathrm{mg}$ to improve pain by $50 \%,{ }^{31}$ EULAR considers that it has a weak recommendation for its use, ${ }^{6,13,30,32}$ the prescription of pregabalin increased in the final treatment, with greater prescription in triple therapy, associated with acetaminophen plus codeine. The safety profile of these two medications limits their use of predominance at the level of the central nervous system due to dizziness, drowsiness, edema and weight gain, ${ }^{16}$ regarding the use of gabapentin, its formulation was scarce and EULAR ${ }^{30}$ does not recommend it. ${ }^{19}$

When comparing combined therapy against monotherapy to determine which is more effective and safe or when one or the other should be formulated, it is difficult to establish, due to the lack of quality studies comparing these therapies. There are studies that compare the management of pregabalin, with amitriptyline or duloxetine showing better pain reduction in combined therapy versus monotherapy, with tolerable adverse effects, the problem is that the majority of studies comparing monotherapy and combined therapy are studies of Low quality, they are very heterogeneous in the combination of medications and the doses used, which makes it difficult to perform meta-analysis on the one hand and does not allow recommendations. ${ }^{33}$

EULAR recommends that pharmacological treatments should be reassessed to ensure the need for continuity and should be prescribed at the lowest effective dose, and ideally for a limited time. ${ }^{14}$ Regarding safety, reports show that the symptoms were not severe, with neurological symptoms (drowsiness and dizziness), gastrointestinal symptoms (diarrhea, vomiting, nausea and epigastralgia), only tramadol presented an episode of tachycardia, the dose is unknown.

The reports were scarce it is not known if it is why they were not presented or why they were not reported, if the cause is for not reporting in the medical records, this may be due to the lack of an institutional pharmacovigilance program, the subreport may occur for different reasons among them they are; 1. Failure to report these by treating doctors, 2 . Due to the lack of therapeutic monitoring, 3 . Lack of compliance with therapy, may be related to non-adherence to medical management, which is very common in patients with FM. A study of Pharmacoepidemiology reported that this is due to: a) a bad doctor-patient relationship, b) receiving multiple treatment for a long period of time and c) does not meet the patient's expectations for medical treatment. ${ }^{34}$ Or because the treatment was not dispensed in the pharmacy.

You can find in this study, prescriptions that increase the risk of adverse events given by interactions given by the pharmacological therapy established for fm, among them we find duloxetine with tramadol which can increase the risk of serotonergic syndrome, it can also increase the risk of seizures not related to this syndrome, most likely if you are elderly. ${ }^{35}$

The combination of amitriptyline and tramadol, increases the risk of seizures, in susceptible people such as older adults, in withdrawal and / or conditions that affect the central nervous system, ${ }^{35}$ codeine and tramadol can cause seizures, respiratory distress, coma and even death, ${ }^{35}$ the use of duloxetine with pregabalin increases adverse effects such as dizziness, drowsiness, confusion and difficulty concentrating, ${ }^{35}$ the combination of Hydrocodone and pregabalin increases side effects such as dizziness, drowsiness, confusion and difficulty concentrating, impaired thinking judgment and motor coordination, ${ }^{35}$ the association of amitriptyline and pregabalin increases side effects such as dizziness, drowsiness, confusion and difficulty concentrating, impaired thinking judgment and motor coordination, ${ }^{35}$ use Simultaneous tramadol and pregabalin causes dizziness, drowsiness, confusion and difficulty concentrating. Gold of thought, judgment and motor coordination, ${ }^{35}$ the use of codeine and pregabalin increases the effects of dizziness, drowsiness, confusion and difficulty concentrating, impaired thinking, judgment and coordination, ${ }^{35}$ the association of codeine and Amitriptyline increases the risk of serotoninergic syndrome ${ }^{35}$ the combination of Hydrocodone and duloxetine increases the risk of serotoninergic syndrome, ${ }^{35}$ the combination of codeine and duloxetine, duloxetine may reduce the effectiveness of codeine. ${ }^{35}$

The risk of therapeutic duplication, when combining drugs that affect the central nervous system (duloxetine, pregabalin, fluoxetine and tramadol) should not be more than three the same as analgesics, opioids can only be combined two and antidepressants should only be used one. ${ }^{35}$ 
Another important aspect is the consumption of opioids, which currently presents an epidemic in the use of opioid in the management of chronic non-cancer pain, this increases the risk of abuse and fatal adverse events, on the other hand this same phenomenon is being observed with the anticonvulsants there is a tendency of increase in its prescription and consumption in Spain and pregabalin and gabapentin predominantly, the majority of formulations are UNIRS, combined with opiates, benzodiazepines and psychotropics, increasing adverse effects, with greater danger in the geriatric population..$^{29}$

It is important to establish that the problem of FM is that its main symptom is pain, and pain is associated with suffering, it is a complex and multidetermined phenomenon, the result of the interaction between sensory, emotional, cognitive and behavioral factors. Which makes the treatment difficult and requires multiple approaches, due to heterogeneity in the FM population, an important component that objectivity is the measurement of the numerical pain scale although it is subjective, it is a reliable method. ${ }^{36}$ This scale allows us to quantify how much pain the patient experiences. In this study, the perception of pain tends to increase over time, which is consistent with that reported in the literature. ${ }^{16}$ This would indicate a therapeutic failure in $79 \%$ of cases, showing very poor efficacy of pain control medications.

The associated symptoms in FM reported in the literature increase and decrease over time, but rarely remit completely, ${ }^{24}$ which allow to classify the severity of this syndrome, the symptoms found in this study, are not different from those reported in Insomnia literature is the most reported, followed by sadness, anxiety and gastrointestinal disorders, little memory impairment, fatigue and weakness were reported, ${ }^{37,38}$ symptoms that are related to increased pain, and severity of FM are; insomnia which is affected by high levels of stress and catastrophization, ${ }^{38}$ fatigue ${ }^{3}$ and depression. ${ }^{24}$

The presence of these symptoms for 3 months or more causes the patient to lose his ability to work and increase his disability. ${ }^{17}$ It is noteworthy that the report of these symptoms was scarce in the medical records, despite their importance in this pathology, and the improvement or not of these with the pharmacological treatment was not evaluated, which is a criterion to continue or suspend the therapy, this may be due to the lack of knowledge of the latest diagnostic criteria of FM (2016) and how to evaluate the impact of the disease and the non-unification of the guidelines and / or pharmacological treatment guidelines.

EULAR recommends that in case of depression related to pain, anxiety, catastrophism, excessively passive or active coping, management should be given as a first line psychopharmacological therapy, another option is cognitive behavioral therapy, ${ }^{14}$ which should be carried out by staff trained for chronic pain management. FM is characterized by being associated with other comorbidities, which are expected for this type of population towards the elderly, in our study the pathologies associated with pain, with a higher percentage were rheumatoid arthritis, osteoarthritis, low back pain and myofascial syndrome. The association of FM and myofascial syndrome is due to muscular retractions due to the anticipation of movements that lead to a greater perception of pain. ${ }^{23}$

It has been seen that the association of fibromyalgia and arthritis, worsens the quality of life, and have higher pain thresholds compared to patients who have rheumatoid arthritis and do not have fibromyalgia, ${ }^{36}$ osteaoatrosis, discopathy and low back pain as well are prevalent in FM, the pathologies that do not increase the perception of pain, but are associated, are metabolic diseases such as diabetes and hypothyroidism and cardiovascular diseases such as hypertension and heart failure. ${ }^{39}$ Although these pathologies were found, in the study population their report was scarce.

Affection plays an important role in FM, due to the high association of this syndrome with mental illnesses, where depression predominates, it is debatable whether this union is due to cause or effect, the two entities share the decrease in serotonin and norepinephrine, which plays an important role in affection and pain pathway, as the etiology of FM is unknown, it is postulated that the origin of this is due to an alteration of the immune system, while depression is due to an alteration of serotonin ", which would establish a difference between the two, others postulate that it is because of cause and effect that one leads to the other ${ }^{38}$ from the biopsychosocial perspective, physical or psychological stress, associated with a genetic predisposition are factors that can be triggers of the pathology, ${ }^{40}$ this background causes different FM subtypes to occur, because they are predisposing, precipitating and perpetual factors, where inter personality and psychological commitment also act, ${ }^{40}$ in this study it is found that in the affective part the greatest commitment is anxiety and depression disorder, followed by depressive disorder, pathologies associated with stress or personality are found in a lower percentage, which share a pathophysiological basis with $\mathrm{FM}^{40}$ in $30 \%$ of cases no history of psychiatric disease was reported, it is not possible to state whether this event occurred, due to its omission in the clinical history, or is because these patients do not present any associated psychiatric illness.

Another possible cause or risk factor for the development of FM, is given by traumatic events triggered in childhood, because an association was found between them and FM, although they are not found in all patients with FM, if they can be related to its severity, or its prognosis, the most reported are sexual abuse and physical and/ or verbal abuse, ${ }^{14}$ there are studies that show that childhood adverse experiences, cause an alteration in the pituitary hypothalamus axis due to the constant aggression to which the infant is subjected, causes this axis to remain chronically active, generating chronic stress, which increases cortisol, adrenaline, alters neuroplasticity, causes immunosuppression and alters the biochemistry of the child, this is due to the direct interaction that exists between the hypothalaushippocampus and the amygdala, because these play a role in storing memories, activating the HPA axis and emotions what predisposes to chronic pain, disease, posttraumatic stress and depression. ${ }^{41}$

These antecedents predispose to present higher pain scores and greater refractoriness to the pharmacological treatment, ${ }^{35}$ the reports of these antecedents were few in the clinical histories, where it is not possible to establish if it is why they did not present it or why it was omitted antecedent, and the reports found, were in the psychiatric evaluation and not by the treating service, which makes us think that the ignorance of this item and its impact on FM, was not taken into account for multimodal management, which would indicate that these patients should be given a different pharmacological management to patients who do not have traumatic experiences in childhood, require a multidisciplinary management with pharmacological and nonpharmacological measures from the beginning of treatment.

The estimated costs are the direct costs of pharmacological treatment, from the perspective of the health system, when comparing the costs of the different therapies used in this study, it is found that the costs of triple therapy are the most expensive and the most risky , combined therapy and monotherapy do not differ much from their 
costs, the therapeutic failure of $79 \%$ of patients is striking, since only $21 \%$ of patients have a perception of pain reduction, this shows that The investment made was not profitable, you should look for treatments that are cost effective that allow the patient to experience disease-free times, which improves their quality of life.

On the other hand, the costs could be considerably reduced, by redistributing this saving to multimodal and non-pharmacological therapy to create a complete program such as that recommended by the EULAR guideline for non-pharmacological treatment, based on education for both the patient and the staff health, provide a comprehensive and multidisciplinary management, which must be staggered and individualized, provide physical therapy aimed at strengthening aerobic training, spaces where hydrotherapy can be performed, trained personnel to provide cognitive behavioral therapy, another option is Biofeedback, acupuncture, meditative movement therapies (qigong, yoga, tai chi). ${ }^{14}$ These different options are mostly not available to this population by the health system.

\section{Limitations of the study}

The limitations of this study were given by a small sample, which could be influenced by the lack of ICD 10 code of fibromyalgia in the subnet software, since the unification of the diagnosis could increase the study population. The quality of the information recorded in the medical history also influenced, which could lead to a lack of background reports, adverse reactions, diagnostic criteria for FM and changes in symptoms associated with drug treatment. Finally, the costs incurred were based on the assumption that everything formulated is dispensed to the patient in the pharmacy.

\section{Conclusions}

The formulation of patients with fibromyalgia is not harmonized with the EULAR 2016 guide, because the associated symptoms were not evaluated, for its formulation and only the evaluation of pain was contemplated, the formulation of medications that increase the risks are continued and are The benefits are null, so they should not be used in this population.

In this study there are high consumption trends for opioids, which offer no benefit, tend to increase pain and create dependence, on the other hand, the lowest consumption trend observed was for anticonvulsants (pregabalin, gabapentin), not Despite the association of these with opiates, antidepressants and / or other central nervous system depressants, they create interaction and increase adverse effects. The majority of drugs formulated for the treatment of fibromyalgia are UNIRS, which indicates a lack of evidence, their long-term safety is unknown, a risk is being assumed, without any benefit, and in the absence of therapeutic resources, It is overprescribing, creating an epidemic of indiscriminate use of mostly narcotic medications, depressants that will alert impulses causing dependency problems, abuse and withdrawal syndrome. Therefore, these medications for this pathology could be classified according to VITP as of doubtful or null value.

The pharmacological management focuses on impacting two therapeutic targets, one is to increase the inhibitory mechanisms of the nociceptive system and the other target is to decrease the excitation of the nociceptive system, in order to interrupt the transmission of pain, but the results are not as expected, due because the literature shows little effectiveness, in most patients, and this may be due to the fact that pain requires a comprehensive management that is not only pharmacological, but also the cognitive, cultural, emotional, behavioral and spiritual part, Therefore, it is necessary to cover as much as possible of these aspects to achieve adequate control of symptoms.

The etiology of FM is still unclear, but in the literature there are strong associations that are involved in the development and prognosis of this disease, despite the limited data obtained in this study, if it can be observed, which is consistent with what has been reported, such as the association of cardiovascular, metabolic, rheumatological diseases, and childhood abuse, which, when associated with FM, tend to cause greater deterioration in the quality of life and increase refractoriness to drug treatment.

The perception of pain given by the numerical pain scale shows moderate to severe pain, despite the pharmacological treatment, this tends to increase and when this decreases the perception of this improvement is low, due to this it is sought to impact the associated symptoms, since by improving sleep, fatigue, depression etc. The pain is being impacted indirectly. In this work it was not possible to see the impact of drug treatment on these associated symptoms, but it is possible to describe the most relevant symptoms, which coincide with what is reported in the literature. The costs of pharmacological treatment can be dramatically reduced, if we take into account that the most expensive drugs are contraindicated in this pathology, multiple therapies are not recommended, which due to their effects may be limiting for the geriatric population, which they predispose them to falls, which would increase their morbidity and mortality. On the other hand, treatment times and therefore the consumption in milligrams can be reduced, if a reassessment is made, to determine if it is necessary to continue with the therapy or dose adjustment.

\section{Recommendations}

a. Doctors and patients should know the FM and the different nonpharmacological and pharmacological treatment guidelines, knowing the benefits and risks.

b. If the decision is made to formulate UNIRS medications, this should be discussed with the patient.

c. Periodically evaluate the effectiveness of the treatment to define whether to continue its use.

d. The information in the medical records should be improved, recording the diagnostic criteria, the numerical scale of the pain, the history, associated symptoms, the treatment and the results of this with the adverse effects. Take into account the prognostic factors and how they might impact them.

e. We must continue with the investigations, carry out clinical studies to evaluate effectiveness, efficacy, combined therapies, monotherapy, economic, epidemiological studies, etc., that allow us to elucidate this pathology, which must be of quality and for a long time. Allow recommendations.

f. The costs that can be reduced in the pharmacological treatment can be invested in creating pharmacovigilance programs and more structured programs that allow carrying out and guaranteeing non-pharmacological measures in each patient individually.

g. It is recommended for future research to create the ICD 10 code for FM, this would facilitate the search, allowing epidemiological, clinical and pharmacoeconomic studies. 


\section{Acknowledgments}

None.

\section{Conflicts of interest}

Authors declare that there is no conflict of interest.

\section{References}

1. Reed M, Herrm M. The Difficulties in Developing and Implementing Fibromyalgia Guidelines. En: Wilke WS, editor. New Insights into Fibromyalgia. InTech; 2012.

2. Http://consultaregistro.invima.gov.co:8082/Consultas/consultas/ consreg_encabcum.jsp.

3. Arnold LM, Crofford LJ, Mease PJ, et al. Patient perspectives on the impact of fibromyalgia. Patient Educ Couns. 2004;73(1):114-120.

4. Ghavidel-parsa B, Bidari A, Maafi AA, et al. The Iceberg Nature of Fibromyalgia Burden: The Clinical and Economic Aspects. Korean $J$ Pain. 2015;28(3):169-176.

5. Fitzcharles MA, Ste-Marie PA, Pereira JX. Fibromyalgia: evolving concepts over the past 2 decades. CMAJ. 2013;185(13):E645-E651.

6. Kwiatek R. Treatment of fibromyalgia. Aust Prescr. 2017;40(5):179183.

7. Wolfe F, Clauw DJ, Fitzcharles MA, et al. Fibromyalgia Criteria and Severity Scales for Clinical and Epidemiological Studies: A Modification of the ACR Preliminary Diagnostic Criteria for Fibromyalgia. $J$ Rheumatol. 2011;38(6):1113-1122.

8. Gomez-Arguelles JM, Maestu-Unturbe C, Gomez-Aguilera EJ. Neuroimaging in fibromyalgia. Rev Neurol. 2018;67(10):394-402.

9. Sluka KA, Clauw DJ. Neurobiology of fibromyalgia and chronic widespread pain. Neuroscience. 2016;338:114-129.

10. Kia S, Choy E. Update on Treatment Guideline in Fibromyalgia Syndrome with Focus on Pharmacology. Biomedicines. 2017;5(2):E20.

11. Wolfe F, Walitt B. Fibromyalgia: A Short Commentary. J Headache Pain Manag. 2016;1(3).

12. Theoharides TC, Tsilioni I, Arbetman L, et al. Fibromyalgia syndrome in need of effective treatments. J Pharmacol Exp Ther. 2015;355(2):255263.

13. Häuser W, Walitt B, Fitzcharles MA, et al. Review of pharmacological therapies in fibromyalgia syndrome. Arthritis Res Ther. 2014;16(1):201.

14. Häuser W, Ablin J, Perrot S, et al. Management of fibromyalgia: Key messages from recent evidence-based guidelines. Polish Arch Intern Med. 2017;127(1):47-56.

15. Macfarlane GJ, Kronisch C, Dean LE, et al. EULAR revised recommendations for the management of fibromyalgia. Ann Rheum Dis. 2017;76(2):318-328.

16. Silverman S, Sadosky A, Evans C, et al. Toward characterization and definition of fibromyalgia severity. BMC Musculoskelet Disord. 2010;11:66.

17. Arnold LM, Cappelleri JC, Clair A, et al. Interpreting effect sizes and clinical relevance of pharmacological interventions for fibromyalgia. Pain Ther. 2013;2(1):65-71.

18. Sáez M, Sanchez N, Jiménez S, et al. Treatment of pain in the elderly non-opioid analgesics. Rev Soc Esp dolor. 2016;23(1):39-44.
19. Macfarlane GJ, Kronisch C, Dean LE, et al. EULAR revised recommendations for the management of fibromyalgia. Ann Rheum Dis. 2017;76(2):318-328.

20. Leopoldino AO, Machado GC, Ferreira PH, et al. Paracetamol versus placebo for knee and hip osteoarthritis. Cochrane Database Syst Rev; 2019.

21. Ngian GS, Guymer EK, Littlejohn GO. The use of opioids in fibromyalgia. International Journal of Rheumatic Diseases. 2011;14(1):6-11.

22. Levine TD, Saperstein DS. Routine use of punch biopsy to diagnose small fiber neuropathy in fibromyalgia patients. Clin Rheumatol. 2015;34(3):413-417.

23. Okifuji A, Gao J, Bokat C, et al. Management of fibromyalgia syndrome in 2016. Pain Manag. 2016;6(4):383-400.

24. Jacobson SA, Simpson RG, Lubahn C, et al. Characterization of fibromyalgia symptoms in patients 55-95 years old: a longitudinal study showing symptom persistence with suboptimal treatment. Aging Clin Exp Res. 2015;27(1):75-82.

25. Cording M, Derry S, Phillips T, et al. Milnacipran for pain in fibromyalgia in adults. Vol. 2017, Cochrane Database of Systematic Reviews. John Wiley \& Sons Ltd; 2015.

26. Walitt B, Urrútia G, Nishishinya MB, et al. Selective serotonin reuptake inhibitors for fibromyalgia syndrome. Group CM, editor. Cochrane Database Syst Rev; 2015.

27. Lunn MP, Hughes RA, Wiffen PJ. Duloxetine for treating painful neuropathy, chronic pain or fibromyalgia. Cochrane Database Syst Rev; 2014.

28. Üçeyler N, Sommer C, Walitt B, et al. Anticonvulsants for fibromyalgia. En: Häuser W, editor. Cochrane Database of Systematic Reviews. Chichester, UK: John Wiley \& Sons, Ltd; 2013.

29. Johansen ME. Gabapentinoid Use in the United States 2002 Through 2015Letters. JAMA Intern Med. 2018;178(2):292-294.

30. Üçeyler N, Sommer C, Walitt B, et al. Anticonvulsants for fibromyalgia. Cochrane database Syst Rev. 2017;10(10):CD010782.

31. Bhusal S, Diomampo S, Magrey MN. Clinical utility, safety, and efficacy of pregabalin in the treatment of fibromyalgia. Drug Healthc Patient Saf. 2016;8:13-23.

32. Littlejohn G, Guymer E. Modulation of NMDA Receptor Activity in Fibromyalgia. Biomedicines. 2017;5(2):E15.

33. Thorpe J, Shum B, Moore RA, et al. Combination pharmacotherapy for the treatment of fibromyalgia in adults. Cochrane Database of Systematic Reviews. John Wiley \& Sons, Ltd; 2018.

34. Sewitch MJ, Dobkin PL, Bernatsky S, et al. Medication non-adherence in women with fibromyalgia. Rheumatology. 2004;43(5,1):648-654.

35. Drug Interaction Report. drugs.com.

36. Levy O, Segal R, Maslakov I, et al. The impact of concomitant fibromyalgia on visual analogue scales of pain, fatigue and function in patients with various rheumatic disorders. Clin Exp Rheumatol. 2016;34(2 Suppl 96):S120-S124.

37. Guymer EK, Littlejohn GO, Brand CK, et al. Fibromyalgia onset has a high impact on work ability in Australians. Intern Med J. 2016;46(9):1069-1074.

38. Gormsen L, Rosenberg R, Bach FW, et al. Depression, anxiety, healthrelated quality of life and pain in patients with chronic fibromyalgia and neuropathic pain. Eur J Pain. 2010;14(2):127.e1-127.e8. 
39. Demirbag BC, Bulut A. Demographic characteristics, clinical findings and functional status in patients with fibromyalgia syndrome. $J$ Pak Med Assoc. 2018;68(7):1043-1047.

40. Gonzalez B, Baptista TM, Branco JC, et al. Fibromyalgia characterization in a psychosocial approach. Psychol Health Med. 2015;20(3):363-368.
41. The Chronic Stress of Poverty: Toxic to Children. The Shriver Report; 2014. 